\title{
LDL subclasses in IDDM patients: relation to diabetic nephropathy
}

\author{
S. Lahdenperä ${ }^{1}$, P.-H. Groop ${ }^{2}$, M.Tilly-Kiesi ${ }^{1}$, T.Kunsi ${ }^{1}$, T. G. Elliott ${ }^{2}$, G. C. Viberti ${ }^{2}$, M.-R. Taskinen ${ }^{1}$ \\ ${ }^{1}$ Third Department of Medicine, University of Helsinki, Helsinki, Finland \\ ${ }^{2}$ Unit for Metabolic Medicine, UMDS, Guy's Hospital, London, UK
}

\begin{abstract}
Summary To answer the question whether the elevation of LDL-cholesterol in IDDM patients with incipient and established diabetic nephropathy is accompanied by changes in LDL size or composition, we studied distribution of LDL particles in 57 normoalbuminuric [AER 7 (1-19) $\mu \mathrm{g} / \mathrm{min}$, median and range], in 46 microalbuminuric [AER $50(20-192) \mu \mathrm{g} / \mathrm{min}$ ] and in 33 proteinuric [AER $422(233-1756) \mu \mathrm{g} / \mathrm{min}$ ] IDDM patients as well as in 49 non-diabetic control subjects with normoalbuminuria. The three diabetic groups were matched for duration of diabetes and glycaemic control. The mean particle diameter of the major LDL peak was determined by nondenaturing gradient gel electrophoresis. Composition and density distribution of LDL were determined in the subgroups of each patient group by density gradient ultracentrifugation. Normoalbuminuric IDDM patients had larger LDL particles than non-diabetic control subjects $(260 \AA$ vs $254 \AA, p<0.05)$. LDL particle diameter was inversely correlated with serum triglycerides in all groups ( $p<0.05$ for normoalbuminuric and $p<0.001$ for other
\end{abstract}

groups). Triglyceride content of LDL was higher in three IDDM groups compared to control group $(p<0.05)$. The elevation of LDL mass in microalbuminuric and proteinuric IDDM groups compared to normoalbuminuric IDDM group ( $p<0.05$ for both) was mainly due to the increment of light LDL (density $1.0212-1.0343 \mathrm{~g} / \mathrm{ml}$ ). There were no significant changes in the density distribution or composition of LDL between the three diabetic groups. In conclusion the increase of LDL mass without major compositional changes suggests that the elevation of LDL in incipient and established diabetic nephropathy is primarily due to the increased number of LDL particles. The prevalence of atherogenic small dense LDL particles in IDDM patients with microalbuminuria and proteinuria is closely dependent on plasma triglyceride concentration. [Diabetologia (1994) 37:681-688]

Key words IDDM, diabetic nephropathy, microalbuminuria, proteinuria, lipid metabolism, small dense LDL
Cardiovascular disease is the major cause of excess morbidity and mortality among IDDM patients [1-4]. In particular the patients with diabetic nephropathy are at high risk of cardiovascular disease and have a relative

Received: 21 October 1993

and in revised form: 3 January 1994

Corresponding author: Professor M.-R. Taskinen, Third Department of Medicine, University of Helsinki, Haartmaninkatu 4, FIN-00290 Helsinki, Finland

Abbreviations: AER, urinary album excretion rate; CETP, cholesteryl ester transfer protein; IDDM, insulin-dependent diabetes mellitus; $\mathrm{CHD}$, coronary heart disease; ApoB, apolipoprotein B. mortality 30-times higher than patients without nephropathy $[5,6]$. Recently, microalbuminuria perse has been recognized as a risk marker for CHD mortality in both NIDDM and non-diabetic populations [7-8]. In IDDM patients microalbuminuria predicts the development of clinical proteinuria but so far the impact of microalbuminuria on CHD mortality in IDDM has been evaluated only in small groups [9]. The link between dyslipidaemias and diabetic nephropathy is commonly recognized, and lipid abnormalities may contribute to the excess $C H D$ risk although the quantitative and differential impact of the several risk factors present in these patients is not established. Several studies have reported elevations of serum total and LDL cholesterol, trigly- 
Table 1. Clinical characteristics of the IDDM patients with

\begin{tabular}{lllll}
\hline & Normoalbuminuria & Microalbuminuria & Proteinuria & Healthy subjects \\
\hline$n$ & 57 & 46 & 33 & 49 \\
Sex $(\mathrm{M} / \mathrm{F})$ & $29 / 28$ & $31 / 15$ & $17 / 16$ & $27 / 22$ \\
Age (years) & $36.0(18.9-61.4)$ & $38.4(21.9-61.4)$ & $38.0(22.9-56.0)$ & $35.6(24.9-67.0)$ \\
Duration of IDDM (years) & $22.0(7.7-43.2)$ & $22.9(7.7-46.5)$ & $25.0(13.5-41.0)$ & \\
BMI (kg/m $\left.{ }^{2}\right)$ & $23.6(19.1-34.0)$ & $24.2(19.1-31.6)$ & $24.3(20.6-31.5)$ & $24.1(17.7-34.9)$ \\
HbA $1 \%$ (\%) & $9.2(6.4-13.5)$ & $9.8(7.6-14.2)$ & $9.8(7.6-13.3)$ & $6.10(4.80-7.00)$ \\
AER $(\mu \mathrm{gg} / \mathrm{min})$ & $7(1-19)$ & $50(20-192)$ & $422(223-1756)$ & $4(0-9)$ \\
Insulin dose (IU) & $45(22-104)$ & $48(20-96)$ & $46(20-122)$ & \\
Systolic BP $(\mathrm{mmHg})$ & $128(90-157)^{\mathrm{a}}$ & $132(100-200)^{\mathrm{b}, \mathrm{c}}$ & $147(107-188)^{\mathrm{c}, \mathrm{d}}$ & $118(93-145)$ \\
Diastolic BP $(\mathrm{mm} \mathrm{Hg})$ & $76(47-95)$ & $80(61-115)^{\mathrm{a}}$ & $85(59-108)^{\mathrm{c}, \mathrm{d}}$ & $75(60-95)$ \\
\hline
\end{tabular}

${ }^{a} p<0.05$ and ${ }^{c} p<0.001$ vs healthy subjects; ${ }^{b} p<0.05$ and ${ }^{\mathrm{d}} p<0.001$ vs normoalbuminuric IDDM patients

Values are medians (range)

ceride and apoB concentrations in IDDM patients with established nephropathy and variable changes have also been found in IDDM patients with microalbuminuria [10-13]. Whether the changes of lipids and lipoproteins correlate with the degree of albuminuria remains however uncertain $[10,12,14]$.

So far much of the attention has been focused on the concentrations of serum lipids and lipoproteins neglecting the heterogeneity of lipoprotein particles. LDL consists of a spectrum of particles which vary in their size, density, composition and possibly atherogeneity. Two distinct LDL subclass phenotypes can be distinguished according to LDL particle size distribution separated by gradient gel electrophoresis [15]. Pattern A consists of a major LDL peak with LDL particle diameter greater than $255 \AA$ and of minor peak with smaller particle size [15]. In pattern $B$ the diameter of the major LDL peak is equal to or less than $255 \AA$ [15]. A predominance of the small, dense LDL particles which occurs in pattern B is considered to be highly atherogenic [15-17]. Growing evidence indicates that serum triglyceride concentration is one important determinant of structural properties of LDL [18-21]. Several studies have reported that decrease of LDL particle size, increased density and abnormal composition of LDL are associated with elevated VLDL concentrations [18-23]. As this could be a feature in the dyslipidaemia of IDDM patients with kidney disease we investigated in the present study the LDL particle size, density distribution and composition in IDDM patients with incipient and established diabetic nephropathy across a large spectrum of AER.

\section{Subjects and methods}

\section{Patients}

This study included a subpopulation of 136 subjects with gradient gel electrophoresis available out of a larger study group of IDDM patients $(n=153)$ who were recruited for a cross-sectional study [Groop P-H, Elliott T, Ekstrand A et al: Lipoprotein abnormalities in Type 1 (insulin-dependent) diabetic patients with renal disease, manuscript in preparation] from the Diabetic Outpatient Clinics of Helsinki University Hospital, Finland and
Guy's Hospital, London, UK. The IDDM patients were selected to have a wide range of AER based on previous annual screening measurements. At the entry the patients were classified into three subgroups according to the median of AER measured by three overnight urine collections. The normoalbuminuric IDDM group consisted of 57 IDDM patients ( 29 men, 28 women) with AER less than $20 \mu \mathrm{g} / \mathrm{min}$. Microalbuminuric IDDM patients (31 men, 15 women) had an AER from 20 to $200 \mu \mathrm{g} / \mathrm{min}$. The proteinuric IDDM group included 33 IDDM patients $(17 \mathrm{men}$, 16 women) with albuminuria over $200 \mu \mathrm{g} / \mathrm{min}$. All IDDM patients had C-peptide values less than $0.05 \mathrm{nmol} / \mathrm{l}$ and they had been treated with insulin since the time of diagnosis. Insulin was being received by 121 IDDM patients as variable regimens of insulin injections and 15 by a pump. Retinopathy, by funduscopic assessment, was observed in 21 of 57 normoalbuminuric, 35 of 46 microalbuminuric and 29 of 33 proteinuric IDDM patients. Clinical signs or history of neuropathy were documented in 22 of 57 normoalbuminuric, 28 of 46 microalbuminuric and 26 of 33 proteinuric IDDM subjects. The control group consisted of 49 healthy subjects ( $27 \mathrm{men}, 22$ women) with normal fasting blood glucose and $\mathrm{HbA}_{1}$ values. All groups were matched for age and $B M I$ values, and the three IDDM groups were matched for duration of diabetes, glycaemic control and insulin dose. All participants had normal renal function and had serum creatinine concentration below $110 \mu \mathrm{mol} / 1$. None of the participants received lipid lowering medication. None of the normoalbuminuric IDDM patients nor any of the healthy subjects were being treated for hypertension. IDDM patients taking diuretics or betablockers were excluded from the study because these drugs may affect the lipid and lipoprotein metabolism [24-26]. Three microalbuminuric IDDM patients were on treatment with ACE inhibitors and one with Ca-channel blockers for hypertension. Six proteinuric IDDM patients were being treated with ACE-inhibitors, one with $\mathrm{Ca}$-channel blocker and two were treated with a combination of ACE-inhibitor and Ca-channel blocker. Three normoalbuminuric, three proteinuric and four healthy subjects were taking oral contraceptives. The participants were asked to abstain from alcohol during the preceding 3 days. Ethical permission for the study was obtained from the Ethics Committees at Guy's Hospital, London, UK and the III Department of Medicine, Helsinki University Central Hospital, Helsinki, Finland. Clinical characteristics of the subjects are shown in Table 1.

\section{Lipids and lipoproteins}

Blood samples were drawn in the morning after an overnight fast. The serum was separated immediately by centrifugation at $3000 \mathrm{rev} / \mathrm{min}$ for $30 \mathrm{~min}$ at $4^{\circ} \mathrm{C}$. The fresh serum samples from 
Table 2. Serum lipid and lipoprotein concentrations of the IDDM patients with

\begin{tabular}{lllll}
\hline & Normoalbuminuria & Microalbuminuria & Proteinuria & Healthy subjects \\
\hline$n$ & 57 & 46 & 33 & 49 \\
Serum cholesterol $(\mathrm{mmol} / \mathrm{l})$ & $4.65(3.52-8.30)$ & $5.33(3.45-8.54)^{\mathrm{d}}$ & $5.36(2.43-8.22)^{\mathrm{d}}$ & $4.97(3.19-7.55)$ \\
Serum triglycerides $(\mathrm{mmol} / \mathrm{l})$ & $0.89(0.49-4.94)$ & $1.07(0.56-2.92)$ & $0.97(0.35-2.20)$ & $0.90(0.37-3.36)$ \\
HDL-cholesterol $(\mathrm{mmol} / \mathrm{l})$ & $1.65(0.89-2.38)^{\mathrm{b}}$ & $1.45(0.96-2.74)^{\mathrm{c}}$ & $1.56(0.95-2.81)$ & $1.38(0.82-2.28)$ \\
LDL-cholesterol $(\mathrm{mmol} / \mathrm{l})$ & $2.65(1.64-5.67)^{\mathrm{a}}$ & $3.00(1.80-5.74)^{\mathrm{d}}$ & $3.03(1.16-5.40)^{\mathrm{d}}$ & $3.10(1.55-4.82)$ \\
VLDL-cholesterol $(\mathrm{mmol} / \mathrm{l})$ & $0.17(0.02-1.46)$ & $0.25(0.04-1.34)$ & $0.22(0.05-0.86)$ & $0.22(0.03-1.07)$ \\
VLDL-triglycerides $(\mathrm{mmol} / \mathrm{l})$ & $0.37(0.10-3.51)$ & $0.47(0.12-2.01)$ & $0.39(0.06-1.53)$ & $0.41(0.07-2.43)$ \\
Apolipoprotein B $(\mathrm{mg} \%)$ & $81.8(53.6-139.5)$ & $86.9(57.6-161.5)$ & $85.3(34.2-143.4)$ & $82.5(34.2-174.2)$ \\
LDL particle diameter $(\AA)$ & $260(238-279)^{\mathrm{b}}$ & $256(232-288)$ & $257(244-283)$ & $254(228-271)$ \\
\hline
\end{tabular}

${ }^{\mathrm{a}} p<0.05$ and ${ }^{\mathrm{b}} p<0.001$ vs healthy subjects; ${ }^{\mathrm{c}} p<0.05$ and ${ }^{\mathrm{d}} p<0.01$ vs normoalbuminuria

Values are medians (range)

Guy's Hospital were kept at $4^{\circ} \mathrm{C}$ and arrived at the laboratory in Helsinki within $24 \mathrm{~h}$. Sequential ultracentrifugation was used to isolate serum lipoprotein fractions (VLDL, LDL and HDL) as previously described [27, 28].

\section{LDL density gradient ultracentrifugation}

Because LDL density gradient ultracentrifugation is extremely laborious and tedious it was performed only in a randomly selected subgroup of patients including 12 normoalbuminuric (3 men, 9 women ), 15 microalbuminuric ( 11 men, 4 women), 12 proteinuric IDDM patients ( 6 men, 6 women), and 13 healthy control subjects ( 9 men, 4 women). After the isolation of VLDL fraction at a density of $1.006 \mathrm{~g} / \mathrm{ml}$ the infranate was adjusted directly to the density of $1.0630 \mathrm{~g} / \mathrm{ml}$ and ultracentrifuged at $35000 \mathrm{rev} / \mathrm{min}$ for $24 \mathrm{~h}$ at $2{ }^{\circ} \mathrm{C}$. The fraction containing both IDL and LDL was removed by tube slicing and dialysed for 8 h against $\mathrm{NaBr}$ solution with a density of $1.0340 \mathrm{~g} / \mathrm{ml}$. The LDL density gradient ultracentrifugation was performed according to the method previously described by Shen et al. [29] with slight modifications. The discontinuous gradient was prepared with $\mathrm{NaBr}$ solutions into Beckman Ultraclear $9 / 16 \times 33 / 1413-\mathrm{ml}$ centrifuge tubes (Beckman Inc., Palo Alto, Calif., USA) and the densities of the sample tubes were controlled directly as previously described [30]. Ultracentrifugation was carried out in a Beckman L8-70 ultracentrifuge with an SW 40TI swinging bucket rotor [ (Cat. No 331302) Beckman Inc.], 26 fractions of $0.5 \mathrm{ml}$ were collected. The four lightest fractions within density 1.0181 to $1.0202 \mathrm{~g} / \mathrm{ml}$ contained IDL and were discarded. The concentrations of cholesterol, free cholesterol, triglycerides, phospholipids and apoB were measured in the remaining 22 fractions (density $=1.0212$ $1.0650 \mathrm{~g} / \mathrm{ml}$ ). The LDL mass in each fraction was calculated by adding together the concentrations of different LDL components. The 11 fractions having mean densities from 1.0212 to $1.0343 \mathrm{~g} / \mathrm{ml}$ were designated light LDL and the 11 fractions with mean densities from 1.0360 to $1.0650 \mathrm{~g} / \mathrm{ml}$ were designated as dense LDL. The LDL peak density is defined as the density of the fraction with the greatest LDL mass.

\section{LDL gradient gel electrophoresis}

Nondenaturing polyacrylamide gradient gel electrophoresis of LDL was performed from frozen serum samples using commercial Pharmacia 2/16 gels (Pharmacia, Uppsala, Sweden) as previously described [31]. The gels were stained with Sudan Black B lipid stain. After the destaining procedure the gels were scanned at $595 \mathrm{~nm}$ with a computer assisted scanning densitometer (Cli- niscan 2; Helena Laboratories, Beaumont, Texas, USA). The mean particle diameter of the main LDL peak was determined by computer performed comparison of the mobility of the sample with that of a calibrated reference LDL preparation run on each gel. Coefficients of variation for intergel and intragel precisions of the used control sample were $1.22 \%$ and $0.98 \%$, respectively.

\section{Laboratory analysis}

The concentration of cholesterol in serum, VLDL, LDL, HDL and LDL fractions and that of triglycerides in serum, VLDL and LDL fractions were determined by an enzymatic colorimetric assay (F Hoffmann La Roche and Co Ltd. Diagnostica, Basle, Switzerland) using an automatic analyser (Cobas Mira, F Hoffmann La Roche and Co Ltd. Diagnostica). The commercial kits were also used to measure the concentrations of free cholesterol (Boehringer Mannheim GmbH, Diagnostica, Mannheim, Germany) and phospholipids (Wako Chemicals, Neuss, Germany) in the LDL fractions isolated by density gradient ultracentrifugation. The concentration of cholesteryl esters was calculated by subtracting the free cholesterol concentration from the total cholesterol concentration. Immunoturbidometric assay (Orion Diagnostica, Espoo, Finland) was used to measure the concentration of apoB in the serum and LDL fractions. Blood glucose concentration was determined by the glucose oxidase method (Auto-Analyzer; Technicon, Tarrytown, NY, USA). Glycosylated haemoglobin $\left(\mathrm{HbA}_{1}\right)$ measurement was carried out by microcolumn chromatography (Isolab, Akron, OH, USA) in Helsinki [32] and by the use of electroendosmosis (Corning Chemical, Palo Alto, Calif., USA) in London. The urine albumin concentration was determined by radioimmunoassay [33].

\section{Statistical analysis}

Analysis of data distribution and comparisons between the groups were performed with BMDP statistical software (University of California Press, 1988) for detailed data description (program 2D) and Kruskall-Wallis analysis of variance (3S). If the Kruskall-Wallis analysis of variance reached statistical significance between four groups, the significances of differences between two groups were assessed with Mann-Whitney nonparametric test (3S). The relationships between the logarithmically transformed variables were estimated with Pearson correlation coefficients. Stepwise regression analysis $(2 \mathrm{R})$ was also performed. 
684
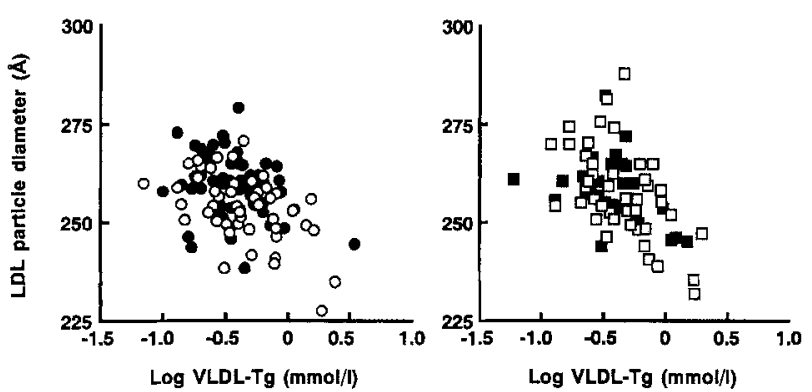

Fig. 1. LDL particle diameter of the major LDL peak vs logarithm of VLDL triglycerides in normoalbuminuric IDDM patients $(\bullet, r=-0.26, p=0.05)$ and healthy control subjects $(O$, $r=-0.51, p<0.001$ ), shown in the left panel, and in microalbuminuric ( $\square, r=-0.55, p<0.001$ ) and proteinuric ( $\boldsymbol{\square}, r=$ $-0.41, p<0.05)$ IDDM patients, shown in the right pane1

\section{Results}

\section{Serum lipids and lipoproteins}

Serum total cholesterol concentration was increased in both microalbuminuric and proteinuric IDDM groups by $15 \%$ ( $p<0.01$ for both), compared to normoalbuminuric IDDM patients (Table 2). LDL-cholesterol concentration was elevated by $13 \%$ in microalbuminuric and by $14 \%$ in proteinuric IDDM subjects compared to the normoalbuminuric IDDM group ( $p<0.01$ for both). Note that LDL-cholesterol values in microalbuminuric and proteinuric IDDM groups were similar to the values in the non-diabetic group. Microalbuminuric IDDM patients had 12\% lower HDL-cholesterol concentration than normoalbuminuric IDDM subjects $(p<0.05)$. Concentrations of VLDL-cholesterol, VLDL-triglycerides and apoB did not differ significantly between the four groups (Table 2). Overall female subjects had higher HDL-cholesterol levels than male subjects in each group, but there were no differences in serum total and LDL-cholesterol or apoB concentrations between the sexes (data not shown). Consequently the data from both sexes were pooled for the detailed analyses of LDL.

\section{LDL particle size}

Distribution of LDL particle size and the mean particle diameters of the major LDL peak were determined in each subject. The mean particle diameter of the major LDL peak was significantly larger in the normoalbuminuric IDDM group (260 A) than in the control group $(254 \AA)(p<0.001)$. The respective diameters of the major LDL peaks were $256 \AA$ and $257 \AA$ in microalbuminuric and proteinuric IDDM groups (Table 2). LDL particle diameter was inversely correlated with the logarithm of VLDL-triglycerides in all groups $(p=0.05$ in normoalbuminuric, $p<0.01$ in microalbuminuric,
S. Lahdenperä et al.: LDL subclasses in diabetic nephropathy
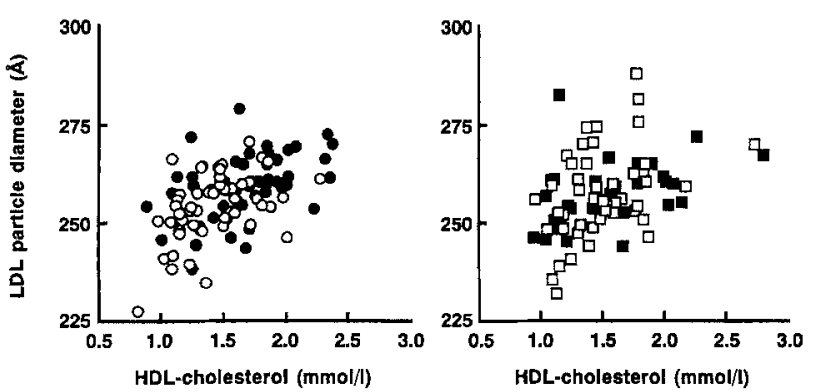

Fig.2. LDL particle diameter of the major LDL peak vs HDLcholesterol concentration in normoalbuminuric IDDM patients $(\bullet, r=0.43, p<0.001)$ and healthy control subjects $(O$, $r=0.47, p<0.001$ ), shown in the left panel, and in microalbuminuric $(\square, r=0.39, p<0.01)$ and proteinuric $(\mathbf{\square}, r=0.41$, $p<0.05)$ IDDM patients, shown in the right panel

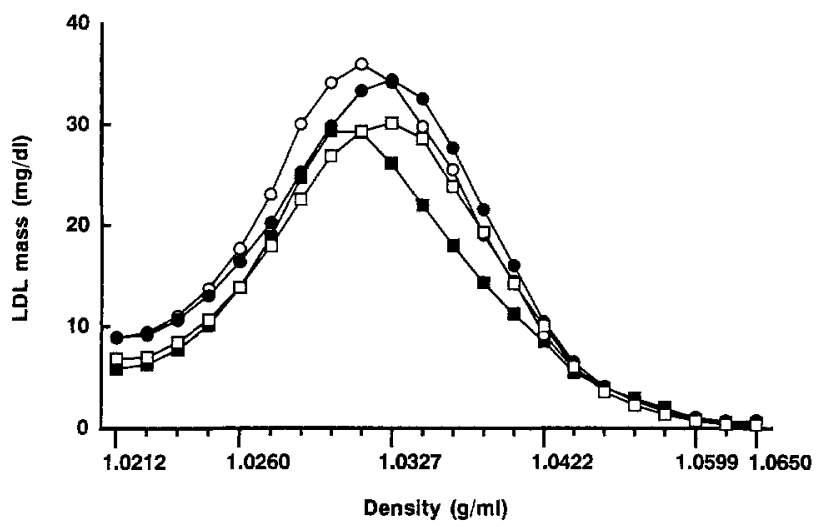

Fig.3. Density distribution of LDL determined by density gradient ultracentrifugation in normoalbuminuric $(\boldsymbol{a})$, microalbuminuric ( $O$ ) and proteinuric ( $)$ IDDM patients, and in healthy control subjects $(\square)$

$p<0.05$ in proteinuric and $p<0.001$ in control subjects, Fig. 1). Note that the range of VLDL-Tg in normoalbuminuric IDDM patients seems to be narrower (range $0.10-0.94 \mathrm{mmol} / \mathrm{l}$ ) than in control subjects (range 0.07 $2.43 \mathrm{mmol} / \mathrm{l})$ if the one outlier with high VLDL-triglycerides $(3.51 \mathrm{mmol} / \mathrm{l})$ is excluded. A significant inverse correlation also existed between the mean diameter of the major LDL peak and the logarithm of serum triglycerides in all groups $(p<0.05$ for normoalbuminuric IDDM group and $p<0.001$ for other groups). A close positive correlation was found between LDL particle diameter and HDL cholesterol concentration in normoalbuminuric IDDM and healthy groups $(p<0.001$ for both, Fig. 2). A similar positive correlation between LDL particle diameter and HDL-cholesterol was observed in microalbuminuric as well as in proteinuric IDDM groups $(p<0.01$ and $p<0.05$, respectively, Fig. 2). When stepwise regression analysis was performed, LDL particle diameter was independently associated with serum triglyceride concentration ( $p<0.001$ for all groups) as well as with HDL-cholesterol concentration $(p<0.05$ for microalbuminuric IDDM group, $p<0.001$ for other groups). 
Table 3. Masses of LDL subfractions and total LDL in the IDDM patients with

\begin{tabular}{llccc}
\hline & Normoalbuminuria & Microalbuminuria & Proteinuria & Healthy subjects \\
\hline$n$ & 12 & 15 & 12 & 13 \\
Sex (M/F) & $3 / 9^{\mathrm{a}}$ & $11 / 4^{\mathrm{c}}$ & $6 / 6$ & $9 / 4$ \\
LDL-cholesterol (mmol/1) & $2.31^{\mathrm{a}}(2.03-4.31)$ & $3.36^{\mathrm{e}}(2.54-5.74)$ & $3.66^{\mathrm{e}}(2.69-5.40)$ & $3.10(1.98-4.70)$ \\
ApoB (mg \%) & $81.6(68.6-98.4)$ & $87.3(58.3-129.2)$ & $98.3(73.7-143.4)$ & $88.3(77.0-124.3)$ \\
LDL particle diameter $(\AA)$ & $265^{\mathrm{b}}(254-279)$ & $256(248-282)$ & $255^{\mathrm{d}}(244-267)$ & $256(228-266)$ \\
LDL mass (mg/dl) & $231.6(182.9-420.1)$ & $334.6^{\mathrm{c}}(185.7-512.8)$ & $319.4^{\mathrm{c}}(202.3-531.6)$ & $282.5(183.2-415.0)$ \\
Light LDL mass (mg/dl) & $165.9(145.2-329.8)$ & $244.9^{\mathrm{c}}(123.9-368.1)$ & $218.8^{\mathrm{c}}(158.8-384.7)$ & $190.1(128.9-316.3)$ \\
Dense LDL mass (mg/dl) & $64.5(26.3-105.7)$ & $75.8(41.6-186.4)$ & $79.7(43.5-180.5)$ & $69.3(31.2-209.2)$ \\
Light LDL cholesterol $(\mathrm{mg} / \mathrm{dl})$ & $71.3(61.1-117.4)$ & $101.7(45.8-163.3)^{\mathrm{d}}$ & $100.8(59.2-170.9)^{\mathrm{c}}$ & $88.5(56.5-138.8)$ \\
Dense LDL cholesterol $(\mathrm{mg} / \mathrm{dl})$ & $25.7(10.8-35.3)$ & $28.2(15.8-87.1)$ & $36.7(13.9-79.0)$ & $30.7(13.0-92.8)$ \\
Light LDL apolipoprotein B $(\mathrm{mg} / \mathrm{dl})$ & $32.9(29.0-47.5)$ & $46.0(24.4-63.2)^{\mathrm{d}}$ & $41.5(31.2-78.1)^{\mathrm{c}}$ & $36.9(27.2-55.7)$ \\
Dense LDL apolipoprotein B (mg/dl) & $14.9(6.0-20.8)$ & $15.1(9.4-37.6)$ & $17.7(8.4-34.7)$ & $15.8(7.9-46.7)$ \\
LDL peak density $(\mathrm{g} / \mathrm{ml})$ & 1.0298 & 1.0312 & 1.0327 & 1.0327 \\
\hline
\end{tabular}

${ }^{\mathrm{a}} p<0.05$ and ${ }^{\mathrm{b}} p<0.01$ vs healthy subjects; ${ }^{\mathrm{c}} p<0.05,{ }^{\mathrm{d}} p<0.01$ and ${ }^{\mathrm{e}} p<0.001$ vs normoalbuminuria Values are medians (range)

Light LDL, LDL with density $1.0212-1.0343 \mathrm{~g} / \mathrm{ml}$; Dense LDL, LDL with density $1.0360-1.0650 \mathrm{~g} / \mathrm{ml}$

Table 4. Percentage distribution of cholesterylesters, free cholesterol, triglycerides, phospholipids and apolipoprotein B in the LDL separated by density gradient ultracentrifugation in IDDM patients with

\begin{tabular}{lllll}
\hline & Normoalbuminuria & Microalbuminuria & Proteinuria & Healthy subjects \\
\hline$n$ & 12 & 15 & 12 & 13 \\
Cholesterylesters & $28.5(22.6-31.7)^{\mathrm{a}}$ & $29.0(14.3-31.9)$ & $27.8(23.7-33.0)$ & $31.8(26.4-34.8)$ \\
Free cholesterol & $13.1(10.2-14.4)$ & $13.4(8.2-30.0)$ & $11.5(8.8-21.8)$ & $13.0(8.9-15.8)$ \\
Triglycerides & $12.1(6.6-15.6)^{\mathrm{b}}$ & $11.6(3.1-18.1)^{\mathrm{a}}$ & $11.4(6.4-18.4)^{\mathrm{a}}$ & $7.5(4.7-15.2)$ \\
Phospholipids & $27.1(23.3-42.4)$ & $27.3(18.3-44.5)$ & $26.3(23.2-38.9)$ & $28.0(24.9-30.4)$ \\
Apolipoprotein B & $19.6(12.7-23.5)$ & $19.7(15.2-21.2)$ & $19.7(15.2-21.4)$ & $20.4(12.6-22.3)$ \\
\hline
\end{tabular}

${ }^{\mathrm{a}} p<0.05$ and ${ }^{\mathrm{b}} p<0.01$ vs healthy subjects

Values are medians (range)

\section{LDL density}

LDL density distribution profiles of the four subgroups are presented in Figure 3. Although the profiles were similar, LDL peak densities displayed a shift to higher density with increasing albuminuria. In both microalbuminuric and proteinuric IDDM subjects total LDL mass was elevated ( $p<0.05$ for both) compared to normoalbuminuric IDDM subjects. Examination of LDL subclasses revealed that the absolute increase of LDL mass was mainly due to that of light LDL (Table 3 ). Concentration of apoB in the light LDL was increased in microalbuminuric and proteinuric IDDM groups by $40 \%$ and $26 \%$, respectively, compared to normoalbuminuric IDDM group $(p<0.01$ and $p<0.05$, respectively, Table 3). Also the cholesterol concentration in the light LDL was higher in microalbuminuric and proteinuric IDDM patients than in normoalbuminuric IDDM patients $(p<0.01$ and $p<0.05$, respectively, Table 3$)$. In the control group light LDL represented $72 \%(43-87 \%)$ of LDL mass and that of dense LDL only $28 \%(13-57 \%)$. Relative percentages of light and dense LDL subfractions from the total LDL mass did not differ between the four groups $(74 \%$ and $26 \%$ in normoalbuminuric, $76 \%$ and $24 \%$ in microalbuminuric and $74 \%$ and $26 \%$ in proteinuric IDDM group).

\section{LDL composition}

The percentage of cholesterylesters in LDL was decreased but that of triglycerides was increased in normoalbuminuric IDDM patients compared to that of healthy control subjects ( $p<0.05$ and 0.01 , respectively, Table 4). The percent content of triglycerides was increased also in microalbuminuric and proteinuric IDDM patients compared to the non-diabetic control group ( $p<0.05$ for both, Table 4$)$. The triglyceride to apoB weight ratio was significantly higher in the three diabetic groups than in the group of healthy control subjects $[0.60(0.39-1.01), 0.56(0.15-1.08)$ and 0.58 $(0.31-0.97)$ vs $0.37(0.21-1.20), p<0.05$ for all]. Cholesteryl esters and free cholesterol to apoB as well as cholesteryl ester to triglyceride weight ratios were similar in all groups (data not shown). Besides triglyceride enrichment in diabetic groups there were no other significant differences in the composition of LDL between the four groups (Table 4).

\section{Discussion}

Dyslipidaemia in our IDDM patients with both microalbuminuria and moderate proteinuria manifested as elevations of serum total and LDL cholesterol concen- 
trations and lowering of HDL-cholesterol concentration. Our results are consistent with previous observations on serum lipids and lipoproteins in diabetic nephropathy [10-13]. The fact that the elevations in serum total and VLDL-triglycerides and in apoB concentrations in microalbuminuric and proteinuric IDDM subjects were not as clear as in some other studies probably reflects the fact that proteinuria was moderate in our proteinuric IDDM group.

In this study we found that in normoalbuminuric IDDM patients LDL particles were larger and less dense than in non-diabetic control subjects. This change together with lowering of LDL mass can be considered to be anti-atherogenic. In IDDM patients with micro- and macroalbuminuria LDL particle diameters of the major LDL peak were comparable to those in non-diabetic subjects. In this study LDL particle size was closely correlated with serum total and VLDL-triglycerides as well as with HDL-cholesterol concentration. More bouyant LDL in normoalbuminuric IDDM patients is thus explained by concomitant changes of VLDL and HDL as compared to nondiabetic subjects. Overall, our data agree with recent evidence that the prevalence of small dense LDL is related to hypertriglyceridaemia and decrease of HDLcholesterol concentration [22,34]. It is hypothesized that a high concentration of VLDL-triglycerides causes an increase in CETP-mediated transfer of cholesteryl esters and triglycerides between VLDL and LDL [18]. Triglyceride-enriched LDL serves as a good substrate for hepatic lipase, which hydrolyses LDL-triglycerides resulting in formation of small dense LDL particles [35]. Therefore, the clinical relevance is that hypertriglyceridaemia in IDDM patients with nephropathy could be associated with preponderance of small dense LDL, which is acknowledged to be a risk factor for CHD. The increased atherogeneity of small dense LDL is assumed to be due to its altered catabolism as well as to its increased susceptibility to oxidation [3639]. On the other hand, there is growing evidence that the LDL subclass pattern is also genetically determined although the exact magnitude of genetic influence on the variation of LDL size is unclear [40]. Also environmental factors such as diet, exercise and use of $\beta$ adrenergic blockers contribute to alterations of LDL particle size $[34,41,42]$.

Abnormalities in the composition of LDL in normoalbuminuric IDDM patients ie. decreased cholesterylesters and increased triglyceride percent content, are consistent with results reported by James and Pometta [43]. However, the increase of triglycerides in the LDL of IDDM patients was not observed by Bagdade et al. [44]. Chapman et al. [45] have shown that increased density and small particle size of LDL are connected with higher percentage content of apoB, lowered cholesteryl ester content and decreased ratio of lipid to apoB in LDL. In line with this observation the total lipid to protein ratio of LDL tended to be higher and the LDL peak particle size was larger in our normoalbuminuric diabetic group compared to the nondiabetic control group. In the present study the triglyceride enrichment of LDL persisted also in microalbuminuric and proteinuric IDDM groups. Overall we observed no other compositional changes of LDL between the three diabetic groups. The data is consistent with the study by Winocour et al. [46] who also could not detect any major compositional changes of LDL in nephropathy.

Our data demonstrated an increase of LDL mass in IDDM patients with incipient and established diabetic nephropathy. This increase of LDL occurred primarily in the light LDL which were $48 \%$ and $32 \%$ higher in the microalbuminuric and in the proteinuric IDDM group, respectively, compared to the normoalbuminuric IDDM group. Elevation of dense LDL was less prominent; $18 \%$ in the microalbuminuric and $24 \%$ in the proteinuric IDDM group. However, the relative percentages of light and dense LDL from the total LDL mass did not differ significantly between the three diabetic groups. The elevation of apoB in the light LDL suggests that the number of these LDL particles is increased. This is further supported by the fact that no major compositional changes were found in the LDL between the three diabetic groups. Overall, higher numbers of LDL particles in the circulation indicate increased atherogeneity. Why is LDL mass increased in patients with incipient or established diabetic nephropathy? Dullaart et al. [47] have observed an increased cholesterylester transfer activity in IDDM patients with microvascular and macrovascular complications. In these patients the cholesterylester transfer activity correlated positively with serum total, VLDL and LDL cholesterol as well as with apoB concentrations suggesting a role for CETP in the development of dyslipidaemia in these diabetic complications. In line with this we observed an inverse correlation $(r=-0.42, p<0.01)$ between fasting VLDL triglycerides and esterified cholesterol to triglyceride mass ratio in LDL of our IDDM subjects. Consequently the driving force could be increased cholesterol ester transfer activity. On the other hand Warwick et al. [48] have shown that proteinuria in non-diabetic patients with the nephrotic syndrome is associated with a defect in LDL catabolism via receptor-mediated pathway. When the disease progresses to gross proteinuria there is also oversynthesis of VLDL, which further exacerbates dyslipidaemia and leads to elevation of triglyceride levels [48]. Other studies implicate that the prodcution of LDL apoB is also increased in the nephrotic syndrome $[49,50]$. These results may not be directly extrapolated to diabetic nephropathy, and kinetic studies need to be performed in patients with diabetic nephropathy.

In conclusion the data suggest that increase of LDL mass in incipient and established diabetic nephropathy is due to the increased number of LDL particles with- 
out compositional changes. In the present study the microalbuminuric and proteinuric IDDM patients had only moderate proteinuria and presented no marked alterations of serum triglycerides. Nonetheless, LDL particle size was closely related to serum triglyceride concentration within each group. The recent recognition of the preponderance of small dense LDL within a range of triglyceride values below the therapeutic target values emphasizes the fact that fasting triglycerides should be maintained as low as possible in IDDM patients with diabetic nephropathy and at high risk of CHD.

Acknowledgements. We are grateful to Drs. R.Weitgasser, A. Mackie, S.Stenman, A. Franssila-Kallunki and A. Ekstrand for help with recruitment and study of patients. We would also like to express our sincere gratitude to Ms. S. Rannikko, Ms. H. Hilden, Ms. K. Runeberg, Ms. L. Lehikoinen and Ms. A.M.Pylkkänen for expert technical help. Work in London was supported by grants from the Wellcome Trust (London) and the Perklen Foundation (Helsinki), while work in Helsinki was supported by the Finnish Academy, the Sigrid Juselius Foundation and Novo Nordisk Insulin Fond. P.-H. Groop was the recipient of a personal grant from the Sigrid Juselius Foundation and the Wellcome Trust.

\section{References}

1. Kannel WB, McGee DL (1979) Diabetes and cardiovascular disease. The Framingham Study. JAMA 241: 2035-2038

2. Green A, Hougaard P (1984) Epidemiological studies of diabetes mellitus in Denmark: 5. Mortality and causes of death among insulin-treated diabetic patients. Diabetologia 26: $190-194$

3. Connell FA, Louden JM (1983) Diabetes mortality in persons under 45 years of age. Am J Public Health 73:1174-1177

4. Krolewski AS, Kosinski EJ, Warram JH, et al. (1987) Magnitude and determinants of coronary artery disease in juvenileonset, insulin-dependent diabetes mellitus. Am J Cardiol 59: 750-755

5. Borch-Johnsen K, Andersen PK, Deckert T (1985) The effect of proteinuria on relative mortality in type 1 (insulin-dependent) diabetes mellitus. Diabetologia 28: 590-596

6. Borch-Johnsen K, Kreiner S (1987) Proteinuria: value as predictor of cardiovascular mortality in insulin-dependent diabetes mellitus. BMJ 294: 1651-1654

7. Mattock M, Morrish N, Viberti GC, Keen H, Fitzgerald A, Jackson G (1992) Prospective study of microalbuminuria as predictor of mortality in NIDDM. Diabetes 41: 736-741

8. Damsgaard EM, Froland A, Jorgensen OD, Mogensen CE (1990) Microalbuminuria as predictor of increased mortality in elderly people. BMI 300: 297-300

9. Messent JWC, Elliott TG, Hill RD, Jarrett RJ, Keen H, Viberti GC (1992) Prognostic significance of microalbuminuria in insulin-dependent diabetes mellitus: A twentythree year follow-up study. Kidney Int 41: 836-839

10. Vannini P, Ciavarella A, Flammini M, et al. (1984) Lipid abnormalities in insulin-dependent diabetic patients with albuminuria. Diabetes Care 7: 151-154

11. Jones SL, Close CF, Mattock MB, Jarrett RJ, Keen H, Viberti GC (1989) Plasma lipid and coagulation factor concentration in insulin dependent diabetics with microalbuminuria. BMJ 298: 487-490
12. Jensen T, Stender S, Deckert T (1988) Abnormalities in plasma concentrations of lipoproteins and fibrinogen in type 1 (insulin-dependent) diabetic patients with increased urinary albumin excretion. Diabetologia 31: 142-145

13. Dullaart RPF, Dikkeschei LD, Doorenbos H (1989) Alterations in serum lipid and apolipoproteins in male type 1 (insulin-dependent) diabetes patients with microalbuminuria. Diabetologia 32: 685-689

14. Holm J, Hemmingsen L, Nielsen N (1989) Serum concentrations of lipids and lipoproteins related to degree of albuminuria and low-molecular weight (tubular) proteinuria in insulin-dependent diabetics. Med Sci Res 17:945-947

15. Austin MA, Breslow JL, Hennekens CH, Buring JE, willet WC, Krauss RM(1988) Low-density lipoprotein subclass patterns and risk of myocardialinfarction.JAMA 260:1917-1921

16. Austin MA, Krauss RM (1986) Genetic control of low-density lipoprotein subclasses. Lancet II: 592-595

17. Austin MA, King MC, Vranizan KM, Krauss RM (1990) Atherogenic lipoprotein phenotype. A proposed genetic marker for coronary heart disease risk. Circulation 82:495-506

18. Deckelbaum RJ, Granot E, Oschry Y, Rose L, Eisenberg S (1984) Plasma triglyceride determines structure-composition in low and high density lipoproteins. Arteriosclerosis 4: $225-231$

19. McNamara JR, Campos H, Ordovas JM, Peterson J, Wilson PWF, Schaefer EJ (1987) Effect of gender, age and lipid status on low density lipoprotein subfraction distribution. Arteriosclerosis 7: 483-490

20. Crouse JR, Parks JS, Schey HM, Kahl FR (1985) Studies of low density lipoprotein molecular weight in human beings with coronary heart disease. J Lipid Res 26: 566-574

21. McNamara JR, Jenner JL, Li Z, Wilson PWF, Schaefer EJ (1992) Change in LDL particle size is associated with change in plasma triglyceride concentration. Arterioscler Thromb 12: $1284-1290$

22. Eisenberger S, Gavish D, Oschry Y, Fainaru M, Deckelbaum RJ (1984) Abnormalities in very low, low and high density lipoproteins in hypertriglyceridemia: reversal toward normal with bezafibrate treatment. J Clin Invest 74: 470-482

23. Cambert P, Bouzerand-Gambert C, Athias A, Farnier M, Lallemant C (1990) Human low density lipoprotein subfractions separated by gradient gel electrophoresis: composition, distribution and alterations induced by cholesterol ester transfer protein. J Lipiḍ Res 31: 1199-1210

24. Weidmann P, de Courten M, Ferrari P (1982) Effect of diuretics on the plasma lipid profile. Eur Heart J 13 [Suppl G]: $61-67$

25. Frishman WH, Furberg CH, Friedewald WT (1984) Betaadrenergic blockade for survivors of acute myocardial infarction. N Engl J Med 310: 830-836

26. Campos H, Genest Jr. JJ, Blijlevens E, et al. (1992) Low density lipoprotein particle size and coronary artery disease. Arterioscler Thromb 12: 187-195

27. Havel JR, Eder HA, Bragdon JH (1955) Distribution and chemical composition of ultra-centrifugally separated lipoproteins in human serum. J Clin Invest 34: 1345-1355

28. LahdenperäS, Tilly-Kiesi M, Vuorinen-Markkola H, KuusiT, Taskinen M-R (1993) Effect of gemfibrozil on low-density lipoprotein particle size, density distribution, and composition in patients with type II diabetes. Diabetes Care 16:584-592

29. Shen MMS, Krauss RM, Lindgren FT, Forte TM (1981) Heterogeneity of serum low density lipoproteins in normal human subjects. J Lipid Res 22: 236-244

30. Tilly-Kiesi M (1991) The effect of lovastatin treatment on low density lipoprotein hydrated density distribution and composition in patients with intermittent claudication and primary hypercholesterolemia. Metabolism 40: 623-628 
31. Nichols AV, Krauss RM, Musliner TA (1986) Nondenaturing polyacrylamide gradient gel electrophoresis. In: Segrest JP, Albers JJ (eds) Methods in enzymology: Plasma lipoproteins. vol. 128. London, Academic Press, pp.417-431

32. Welch SG, Boucher DJ (1978) A rapid micro-scale method for the measurement of haemoglobin $A_{1(a+b+c)}$. Diabetologia 14: 209-211

33. Keen H, Chlouverakis G (1963) An immunoassay for urinary albumin at low concentrations. Lancet 2:913-916

34. Campos H, Blijlevens E, McNamara JR, et al. (1992) LDL particle size distribution: results from the Framingham offspring study. Arterioscler Thromb 12: 1410-1419

35. Zambon A, Austin MA, Brown BG, Hokanson JE, Brunzell JD (1993) Effect of hepatic lipase on LDL in normal men and those with coronary artery disease. Arterioscler Thromb 13: $147-153$

36. Lopes-Virella MF, Sherer GK, Lees AM, et al. (1982) Surface binding, internalisation and degradation by cultured human fibroblasts of low density lipoproteins isolated from type 1 (insulin-dependent) diabetic patients: changes with metabolic control. Diabetologia 22: 430-436

37. Kleinman Y, Eisenberg S, Oschry Y, Gavish D, Stein Y (1985) Defective metabolism of hypertriglyceridemic low density lipoprotein in cultured human skin fibroblasts. J Clin Invest 75: 1796-1803

38. Aviram M, Lund-Katz S, Phillips MC, Chait A (1988) The influence of triglyceride content of low density lipoprotein on the interaction of apolipoprotein B-100 with cells. J Biol Chem 263: 16842-16848

39. Chait A, Brazg RL, Tribble DL, Krauss RM (1993) Susceptibility of small, dense, low-density lipoproteins to oxidative modification in subjects with the atherogenic lipoprotein phenotype, pattern B. Am J Med 94: 350-356

40. Austin M, King M-C, Vranizan KM, Newman B, Krauss RM (1988) Inheritance of low-density lipoprotein subclass patterns: results of complex segregation analysis. Am J Hum Genet 43: 838-846
41. Campos H, Willett WC, Peterson RM, et al (1991) Nutrient intake comparisons between Framingham and rural and urban Puriscal, Costa Rica: associations with lipids, lipoproteins, apolipoproteins, and low density lipoproteins particle size. Arterioscler Thromb 11: 1089-1099

42. Lamon-Fava S, Fischer EC, Nelson NE, et al. (1989) Effect of exercise and menstrual cycle status on plasma lipids, low density lipoprotein particle size, and apolipoproteins. J Clin Endocrinol Metab 68: 17-21

43. James RW, Pometta D (1990) Differences in lipoprotein subfraction composition and distribution between type 1 diabetic men and control subjects. Diabetes 39: 1158-1164

44. Bagdade JD, Ritter M, Subbaiah PV (1991) Accelerated cholesterol ester transfer and abnormal surface lipid composition in type 1 diabetes. Eur J Clin Invest 21: 161-167

45. Chapman MJ, Goldstein S, Lagrange D, Laplaud PM (1981) A density gradient ultracentrifugal procedure for the isolation of the major lipoprotein classes from human serum. J Lipid Res 22: 339-358

46. Winocour PH, Durrington PN, Bhatnagar D, Ishola M, Mackness M, Arrol S (1991) Influence of early nephropathy on very low density lipoprotein (VLDL), intermediate density lipoprotein (IDL), and low-density lipoprotein (LDL) composition. Atherosclerosis 89: 49-57

47. Dullaart RPF, Groener JEM, Dikkeschei LD, Erkelens DW, Doorenbos $\mathrm{H}$ (1989) Increased cholesterylester transfer activity in complicated type 1 (insulin-dependent) diabetes mellitus - its relationship with serum lipids. Diabetologia 32: 14-19

48. Warwick GL, Caslake MJ, Boulton-Jones JM, Dagen M, Packard CJ, Shepherd J (1990) Low-density lipoprotein metabolism in the nephrotic syndrome. Metabolism 39:187-192

49. Joven J, Villabona C, Vilella E, Masana L, Alberti R, Valles M (1990) Abnormalities of lipoprotein metabolism in patients with the nephrotic syndrome. N Engl J Med 323:579-584

50. Vega GL, Grundy SM (1988) Lovastatin therapy in nephrotic hyperlipidemia: effects on lipoprotein metabolism. Kidney Int 33: 1160-1168 